\title{
Using a research laboratory for training students
}

\section{Anna Consortini}

Anna Consortini, "Using a research laboratory for training students," Proc. SPIE 9665, Tenth International Topical Meeting on Education and Training in Optics and Photonics, 96651F (3 June 2007); doi: 10.1117/12.2207522

SPIE Event: Tenth International Topical Meeting on Education and Training in Optics and Photonics, 2007, Ottawa, Ontario, Canada 


\title{
Using a Research Laboratory for training Students
}

\author{
Anna Consortini \\ Universita' degli Studi di Firenze, Dipartimento di Fisica, Polo Scientifico, Via G. Sansone 1, 50019 Sesto Fiorentino - Firenze, Italy, \\ Tel+390554572237, email anna.consortini@unifi.it
}

\begin{abstract}
We present a way to use a Research Laboratory for training students to learn about simple measurements. In most cases, doing research and training students imply conflicting requirements, for instance in a Research Laboratory everything is aligned, some equipment cannot be moved and so on; therefore research and training laboratories are separated. Sometimes however, there is the possibility of using a part or the complete research equipment by the students, without "consequences". As an example, here we describe the way we used a research set up of wave propagation through atmospheric turbulence, devoted to long lasting statistical measurements, to train students in making position measurements and experiments at different levels, starting from beginners up to advanced and $\mathrm{PhD}$ students.
\end{abstract}

\section{1 - Introduction}

Typically, a Research Laboratory is equipped with sophisticated apparatuses devoted to specialized experiments and measurements, which are based on technologically advanced instrumentation, used for long-lasting measurements, with small or no flexibility. In most cases, these apparatuses cannot be used for simple experiments and measurements, like those needed for training students, for many reasons, such as the impossibility of removing the instruments from the experimental set up or the need of time to devote to the students and so on.

Training students, on the contrary, requires flexible instrumentation allowing simple and easy measurements and this is generally made in "on purpose" student laboratories. Therefore, there is the need of equipping training laboratories with suitable apparatuses, which in some cases duplicate some of the research instruments and also increases the financial need.

In the past, we set up an apparatus for "local" research on atmospheric turbulence, more precisely to measure lateral fluctuations of a number of "thin" parallel laser beams after a short (few meters) path in the atmosphere. "Thin beam" means a beam whose diameter is not larger than the smallest dimensions of the inhomogeneities of the turbulence. The use of good HeNe lasers, with small divergence, and the short path allow this requirement to be satisfied for what concerns this paper. The apparatus was made flexible to work both in the laboratory and in the open atmosphere. The need of making measurements in the laboratory also originated by the need of testing different propagation theories in controlled turbulence, to develop methods to characterize turbulence and to validate different methods to measure the parameters of turbulence. Without entering the details of this complex research, here I describe the apparatus and some characteristics which make it suitable for training students in

Tenth International Topical Meeting on Education and Training in Optics and Photonics, edited by Marc Nantel, Proc. of SPIE Vol. 9665, 96651F · @ 2007 SPIE, OSA, IEEE, ICO doi: $10.1117 / 12.2207522$ 
the laboratory to learn how to make "static measurements" and also measurements of fluctuating quantities. One of the advantages for the students is also due to the fact that there are some periods along the year that generally coincide with the students training time, when outside measurements are not made. Training in this laboratory can be at different levels, starting from beginners, which can make simple laboratory experiments, up to $\mathrm{PhD}$ students, which learn how to make sophisticated experiments.

\section{2 - Experimental set up}

The basis of the experimental research is to send a number of parallel laser beams (up to four) through a region of natural, or heater produced, turbulence, to let each beam impinge on a position sensor and to measure a large number of subsequent "instantaneous" positions of each beam on its sensor surface. The data are subsequently elaborated by using statistical programs; the correlations between the fluctuations of the different beams are obtained, which are of interest for the research on turbulence.

The scheme for the horizontal measurements in the laboratory is sketched in Fig 1, where also the menu of different required operations appears.



Fig 1 Scheme of the apparatus

The HeNe lasers are located on a movable support, tripod, heavy enough, but easy to carry. The lasers are fixed on a platform, which can be moved up and down and tilted (in the case of slant path) or adjusted to get a horizontal position.

The length of the path depends on the purposes, and in the Laboratory it can reach about $10 \mathrm{~m}$, typical values are around few meters.

At the end of the path, a system of four sensors and amplifier (UDT OpEye) is located on a wood table which is heavy enough to prevent from vibrations. Each eye is located on a three axis micrometric table to allow centering of the beam. On its turn, the amplifier is connected to the acquisition system, which is located on a wheel table, easy to move. 
The possibility of easily moving the different parts of the apparatus is an important feature for the research activity in the laboratory and, mostly, in the open air, to allow different path lengths.

All the system is equipped to work with a maximum of four lasers and four receivers, while for training the students, and sometimes also for research, two lasers and the two corresponding sensors are only used. This is possible thanks to the flexibility of the apparatus and the corresponding acquisition and elaboration software. From now on, I will mostly refer to the two-beam version, in the case of horizontal propagation, which is the simplest configuration and is suitable for the students to make a number of training experiments.

The buttons in Fig 1 indicate the menu of the software and the different operations that can be performed with the apparatus: for the organization of the measurements, for the data acquisition, and to elaborate the data and present the results. The rate of data acquisition can be decided by the user. Here it is kept to a typical value, of $300 \mathrm{data} / \mathrm{s}$, often used for turbulence measurements

\section{3 - Calibration facility}

The most useful procedure for training students is the first operation: Calibration. Calibration a is software that allows one to align each laser beam and to center it on the corresponding sensor surface.

With reference to Fig 2 t,he main features of this procedure are here described, with some detail, by also pointing out how they are used for the students.



Fig 2 Screen presentation of the calibration of the four sensors 
Each sensor, a so-called lateral effect sensor, has a surface of a square with $1 \mathrm{~cm}$ side. From each side a current is collected whose value depends on the distance of the "intensity baricenter" of the impinging beam from the side. The difference between the currents of the vertical sides, normalized to their sum, gives a quantity proportional to the horizontal coordinate which, in turn, after suitable normalization, gives the horizontal position, $\mathrm{x}$, in $\mathrm{mm}$, with respect to the center of the sensor which is taken as origin of the $\mathrm{x}$ and $\mathrm{y}$ axes.

Analogously the difference between the currents from the horizontal sides gives the vertical position, $y$, while the sum of all the currents allows one to obtain the intensity (more precisely the power density in $\mu \mathrm{W} / \mathrm{cm}^{2}$ ).

Fig. 2 is a representation of the main screen of the Calibration, where the $1 \mathrm{~cm}^{2}$ surfaces of the four basic sensors appear. In this figure, one (low energy) laser is used and therefore one sensor only (OP-EYE A) is active. Here, for each sensor, the range of $\mathrm{x}$ and $\mathrm{y}$ is from $-5 \mathrm{~mm}$ to $5 \mathrm{~mm}$, corresponding to the entire sensor surface. The program also allows one to "zoom" the central $\mathrm{mm}^{2}$, by using the lever at the left top of the screen, as will be seen in the next figure, where the scales will also be displayed on the axes.

The fact that all four sensors appear on the screen is the only not-flexible point of the software. However it is not a problem and is useful to show simultaneously the students the effect of the dark currents from the unused sensors. The numbers appearing near each EYE are values of positions and intensity "averaged" over a number of data, acquired at the decided rate, as will be seen in the next step.

Entering in one active OP-EYE, e.g. A, the program gives information on all the details of the measurement. In Figure 3, where the surface of the sensor appears, only the central part $\left(1 \mathrm{~mm}^{2}\right)$ is selected, as can be seen from the scales of the axes, from $-0.5 \mathrm{~mm}$ to $0.5 \mathrm{~mm}$. In addition to the averages, the corresponding variances are presented. From the intensity one can see that the power of the laser used here is about ten times higher than that of the previous figure.

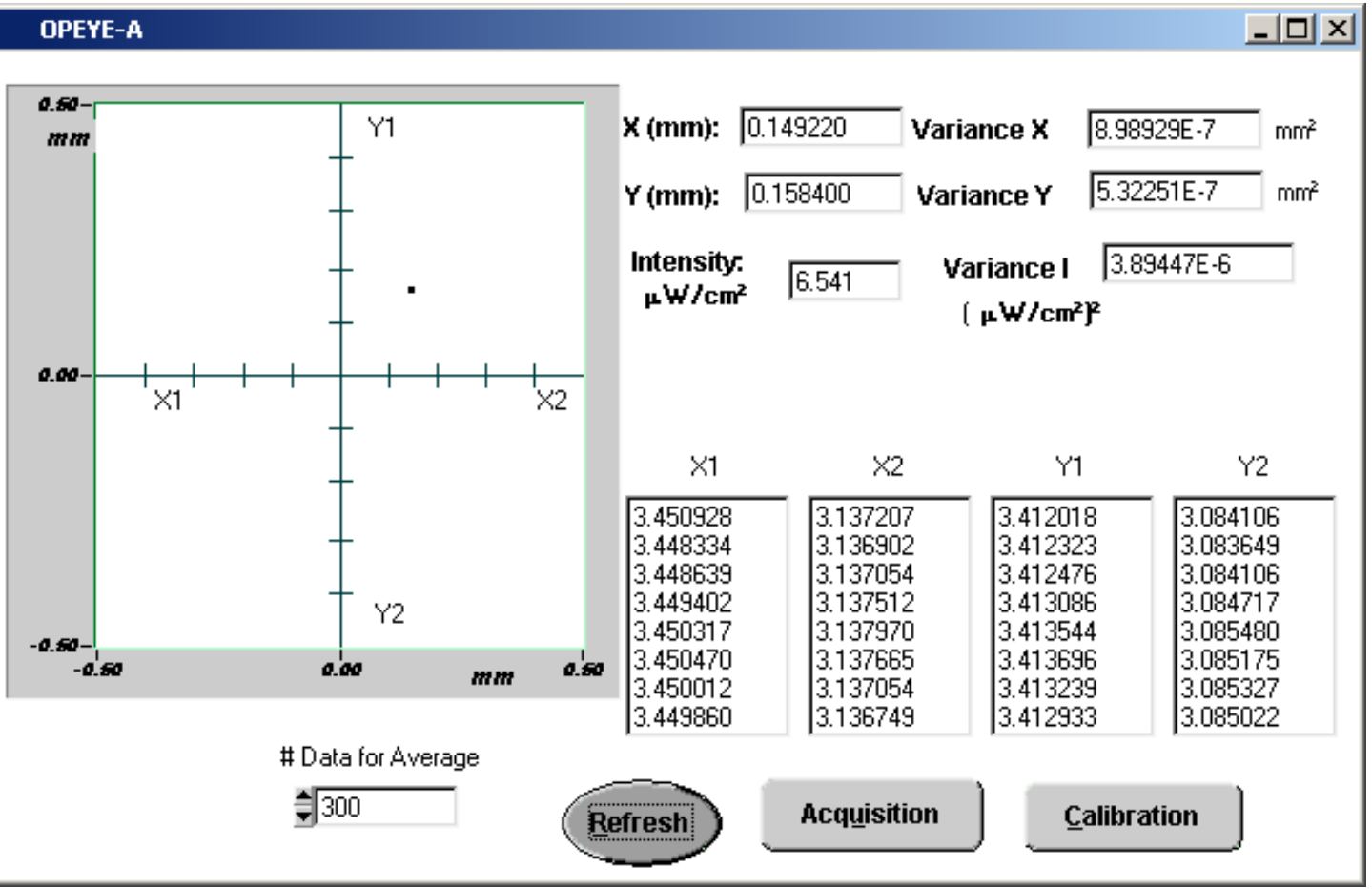

Fig 3 Screen presentation of one sensor calibration at the inner $\mathrm{mm}^{2}$ 
As appears from the small box on the left of the figure, the number of data, used here for the averages, is 300 (it corresponds to one second duration of the measurement according to the decided rate). This number can be chosen starting from a minimum of 10 . When calibrating the apparatus for research measurements, typical values are of some hundreds, while for the students, when they prepare "static" position measurements, 10 data or few tens are sufficient.

The details of the measurement can also be seen on the four bottom columns, each one giving eight subsequent values of the instantaneous currents from the four sides of the sensor surface. They are very useful to show the students how they vary when the laser spot moves on the sensor, for instance when moving the sensor for centering it, and how their difference vanishes when approaching the center.

It is interesting to note that, in the case of static position measurements made by the students, the variances give immediately the "errors" of the measurements to the students. In the research on turbulence, variances of fluctuations and correlation of fluctuations are of different interest and have different meaning because they are fluctuations produced by the turbulence and carry information on turbulence.

\section{4 - Data Acquisition facility}

When the procedure of alignment is completed, one can start the real measurement by using the Acquisition program. As one can see from Fig 4, the Acquisition program allows one to choose the active sensors, the acquisition rate, already mentioned, and the complete duration of the measurement, in seconds.

\section{Data Acquisition}

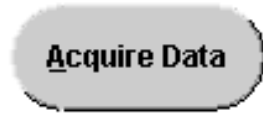

Duration of Measurment in s

$$
600.000000
$$

Rate of acquisition per single opeye (sample/s)

$$
\stackrel{\nabla}{1000}
$$

Time length of an ADC conversion in ms

0.25



Opeye C Opeye D

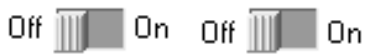

\#Channels actived

4



\section{ns}

.


data/s) and lasting for 5 minutes (300 s). In static situations the duration can be as small as few tens of seconds or one or two minutes, just to give the students the possibility of dealing with a large amount of data. The information about the active sensors is given to the system by means of the four switches at the bottom left of the figure, and the program informs us about the active Channels: four channels for each sensor. It also informs us about the "Time length of an ACD conversion".

There is some other information required by the program, including comments.

The measurement starts by the button Acquire Data. At the beginning of the measurement, an initially green spot, near the button on the screen, becomes red and returns to green at the end. Waiting immobile and silent during one, two or even five minutes is an interesting experience for the students because they fill directly the time passing. At the end of each measurement, the system produces a file "dat", labeled with eight numbers corresponding to month, day, hour and minute of the beginning of the measurement. For instance 11171708.dat is a file made on 11 November at 5:08 pm (17:08 in 24 Hours). No information is directly given about the year, which generally labels an external envelope. With the data file, another initial (.ini) file is produced with the same name containing all the information of the acquisition.

\section{5 - Elaboration programs}

There are several elaboration programs, which were developed along the years. The last and more complete one includes correction for the non-linearity of the sensors and allows one to split the entire duration in evaluation subintervals. For the students we generally use a good previous program, where the evaluation includes the entire measurement duration and the non-linearity is corrected.

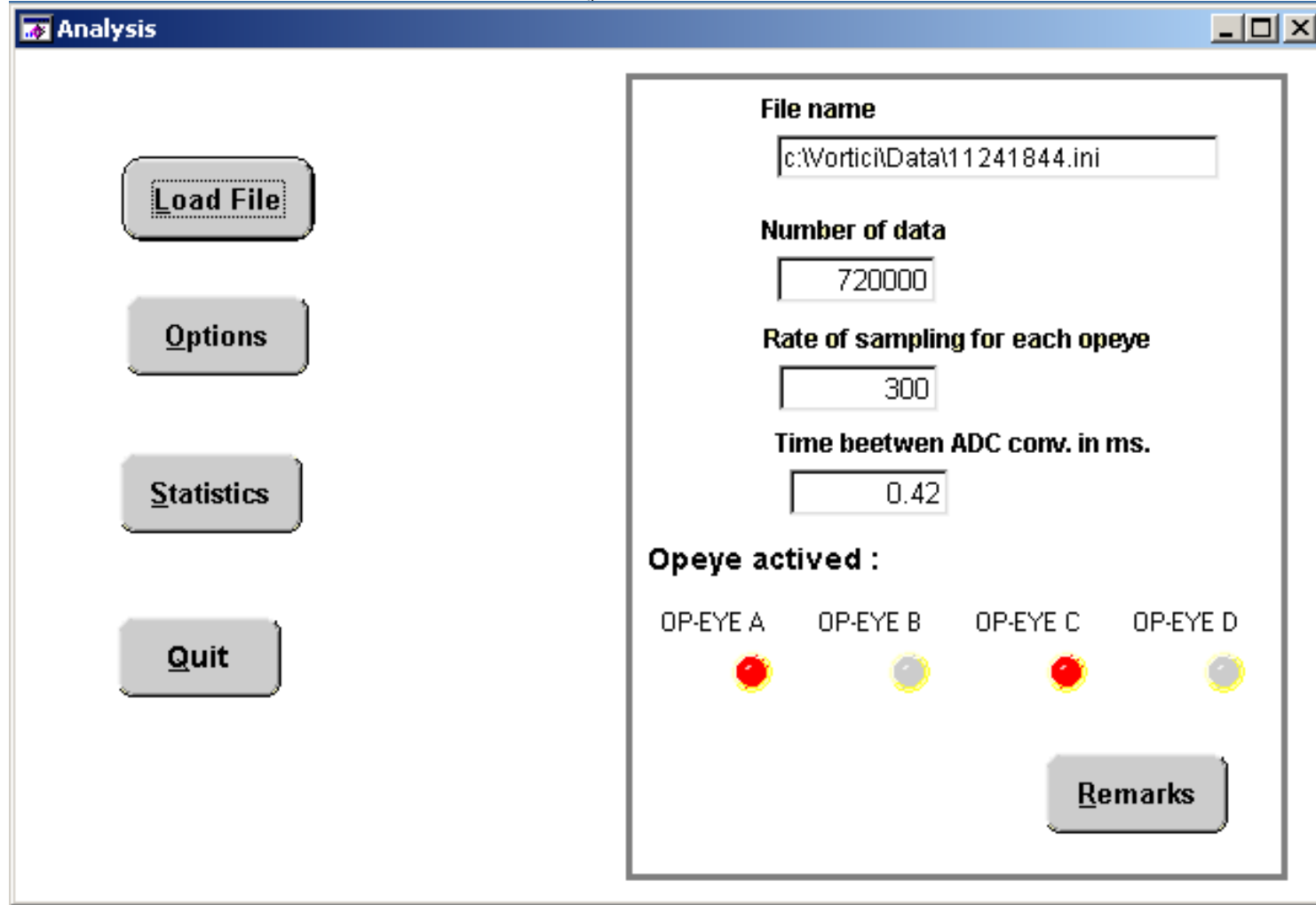

Fig 5 - Menu for the elaboration 
As appears from Fig 5, the first step is to load the file one wants to elaborate: File name. The example is from laboratory training in 2006, when the students of an advanced course measured and elaborated the position fluctuations of two beams, due to turbulence, to the purpose of obtaining the correlation functions of the lateral fluctuations. On the right side of the figure, in addition to the name of the data file, the information from the initial file is represented. Here we know that the data were obtained with two laser beams, impinging on OPEYE A and C respectively, on November 24 at a rate of 300 data/s at h 18:44 (h 6:44 pm). As in the data files the original four currents of each sensor are recorded, the number of data here (720 000) represent the total number of currents recorded from the sensors. The duration of the measurement was $300 \mathrm{~s}=720000:(300 \times 4 \times 2)$. Thinking about how to obtain this time is a very useful exercise for the students.

The Options button allows one to activate also the optional program of the correlations while the Statistics button starts the complete evaluation.

An example of elaboration results is given in Fig 6, which refers to the results of an advanced experiment of a student for her thesis degree (Laurea) in Physics, in 2004. She investigated the effect of wind and heating on an ensemble of four parallel beams in the laboratory, with the beams located at different distances from a system of heaters and fans.

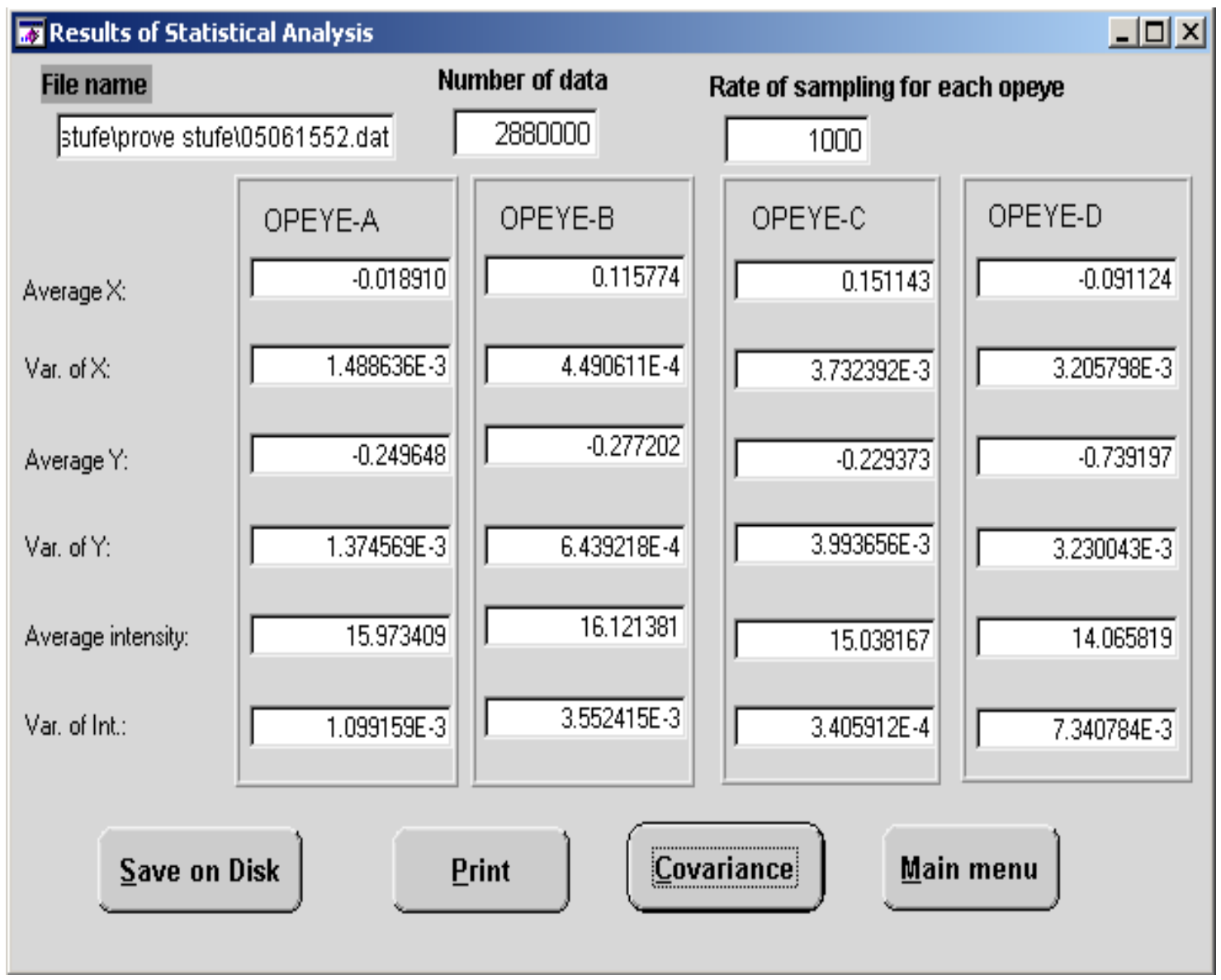

Fig 6 - Example of results

In the figure the first page of the obtained results, as they appear on the computer screen, is represented. A second page, accessible by the Covariance button and showing the correlations results, is not presented here. 
First, information on the date of measurement, number of data and rate of sampling is given. Then a column for each sensor shows averages and variances. All coordinates are in $\mathrm{mm}$, their variances in $\mathrm{mm}^{2}$, the intensity is in $\mu \mathrm{W} / \mathrm{cm}^{2}$ and the intensity variances in $\left(\mu \mathrm{W} / \mathrm{cm}^{2}\right)$. Although the order of the beams is not of concern here, it can be interesting to know that the beam impinging on OPEYE-C was the nearest to the heaters and fans, and then beams D, A and B followed at different spacing.

Another useful program, developed for our research, allows one to plot versus time the instantaneous values of the positions and intensity of the four OPEYEs, by choosing the percentage of data to use, typically $10 \%$. This program is very useful for checking the quality of the measurement. For instance, once, in outside measurements, a fly crossed one of the beams and this was clearly seen on the plot.

\section{6 - Experiments made in training students}

Due to the flexibility of the apparatus, many different training experiments were made and many others are feasible, for students at the different levels. Here there is a list of some experiments already done several times, by using the apparatus:

-Simple position measurements of a "static" source at different source positions. For beginners.

-Measuring the pointing and intensity stability of a HeNe laser, in time, during several hours, typically 8 hours, by making samples at established intervals. For beginners.

-Profiling the Gaussian shape of a laser beam (this requires a pin hole in front of the sensor and, if the beam is not large enough, enlargement by a few meter propagation). Intermediate level.

-Measuring the "apparatus and room noise" on the position of a laser beam after one or two meters path. Intermediate level

-Verification of the effect of intensity instability on position measurements. Advanced level.

- Measuring the non linearity of the sensors outside the inner part, in the horizontal and vertical direction. Intermediate level. An improvement of this experiment, for higher level students, consisted in mapping the non linearity through the entire surface and comparing it with theoretical expectations.

-Measuring the M2 factor of a laser. Advanced level.

-Effect of the "room turbulence" on beam wandering of two parallel beams, when the path is relatively long, and correlation between the wandering of the beams as a function of their separation, to the purpose of deriving information on the dimensions of the turbulent eddies in the room. Very advanced level, $\mathrm{PhD}$.

-Effect of a turbulent layer: measurement of wandering of a beam and comparison with that in the absence of turbulence. Intermediate level. A more advanced experiment consists in measuring the beam variances (and correlations) as a function of the distance of the layer from the sensor, and comparison with the theory.

-Enhancement of fluctuations by double passage of a beam through the same layer of turbulence. PhD level.

At the end, I would like to mention here that, in our research laboratory, students can also make a number of experiments and see a numer of demonstrations, for instance on diffraction and interference, without using the research apparatus. They were not described here.

For the sake of completeness some references on this research are listed here below. 


\section{Acknowledgement:}

I would like to mention that all things that I described here are also the results of the work of a number of students, of different level, some of which also collaborated in the research, and whose interest stimulated me to do this training activity in the past years. In particular, the ABF Corporation of Fig 1 was a joke of three Post Doc students, Agabi, Bezzi, Fusco, who organized, on a PC, the acquisition, previously made by means of Apple Computers. Gianni Paoli, student, and my research collaborator, Claudia Innocenti, made many subsequent improvements in the analysis and elaboration programs. I thank all of them.

\section{References}

1 - A. Consortini and K. A. O'Donnell "Beam wandering of thin parallel beams through atmospheric turbulence", Waves in Random Media 3, S11-S28, 1991.

2 - A. Consortini, G. Fusco, F. Rigal, A. Agabi and Y.Y. Sun "Experimental verification of thin-beam wandering dependence on distance in strong indoor turbulence", Waves in Random Media 7, p.521-529, 1997

3 - A. Consortini, C. Innocenti, G. Fusco and Y.Y. Sun "Double Passage Enhancement of Thin Beam Wandering Through Atmospheric Turbulence", Journal of Modern Optics 48, 1569-1581, 2001

4 - A.Consortini, C.Innocenti, G.Paoli, "Estimate method for outer scale of atmospheric turbulence", Optics Communications 214, 9-14, 2002.

5 - A. Consortini, Y.Y. Sun, C. Innocenti and Z. P. Li "Measuring inner scale of atmospheric turbulence by angle of arrival and scintillation", Optics Communications .216, 19-23, 2003

6 - C. Innocenti and A. Consortini "Estimate of characteristic scales of atmospheric turbulence by thin beams: comparison between the von Karman and Hill-Andrews models", Journal of Modern Optics 51, 333-342, 2004.

7 - C. Innocenti and A. Consortini "Refractive index gradient of the atmosphere at near ground levels", Journal of Modern Optics 52, 671-689, 2005.

8 - Y.Y .Sun, A. Consortini and Z.P. Li "A new method for measuring outer scale of atmospheric turbulence" Waves in Random and Complex Media 17, 1-8, 2007. 